\title{
Importancia de atender el trauma vascular oportunamente y contar con centros de referencia
}

\section{Importance of attending vascular trauma in a timely manner and having reference centers}

\author{
Sandra Olivares-Cruz ${ }^{1}$, Melvin Parada-Guzmán ${ }^{1}$, Beatriz Herrera-Camacho², Jessica I. Islas-Alcalá2, \\ Karla Vargas-González ${ }^{2}$, Verónica Carbajal-Robles ${ }^{1}$ y Dalid R. Nieto-Zepeda ${ }^{1 *}$ \\ ${ }^{1}$ Servicio de Angiología y Cirugía Vascular, Hospital General de México; ${ }^{2}$ Servicio Social, Centro de Salud T-III, José Castro Villagrana. Ciudad de \\ México, México
}

\section{Resumen}

Antecedentes: El trauma vascular se ha incrementado de manera exponencial en los últimos años. Se ha observado que un retraso en el tratamiento impacta directamente en la sobreviva libre de amputación y en diversas complicaciones, por lo que es imperativo otorgar un tratamiento adecuado en un periodo de tiempo breve. Objetivo: Determinar si en los pacientes con diagnóstico de trauma vascular atendidos de manera inicial en el Hospital General de México (HGM), comparados con aquellos que su primer nivel de atención fue un hospital de referencia, presentaron menor número de amputaciones o alguna otra complicación. Material y métodos: Se identificaron los expedientes de los pacientes atendidos en el HGM con el diagnóstico de trauma vascular; se dividieron en dos grupos, los pacientes atendidos de manera inicial en el HGM y aquellos referidos de otras unidades hospitalarias, y se recolectaron las variables de edad, género, comorbilidades, mecanismo de trauma, tiempo transcurrido entre el trauma y el tratamiento definitivo, tipo de reparación vascular, nivel de amputación, insuficiencia renal aguda y mortalidad. Resultados: De los 101 pacientes identificados, se eliminaron 45 expedientes y se incluyeron a 61 pacientes. No hubo diferencia en cuanto a las variables demográficas. Se observó que a la exploración no se palparon pulsos en el $82 \%$ de los pacientes vs. el $57 \%$ de los referidos, al ingreso en el $27 \%$ de los referidos vs. el $3 \%$ de los tratados en el HGM ( $p=0.01)$. Conclusión: Se observó que, en los pacientes referidos, el promedio de tiempo entre el trauma y la atención fue de 7 días. Se incrementó el requerimiento de fasciotomías en estos pacientes, así como un incremento en los tiempos quirúrgicos, por lo que se propone realizar una referencia temprana a un centro de tercer nivel.

Palabras clave: Trauma vascular. Complicaciones. Atención oportuna.

\section{Abstract}

Background: Vascular trauma has increased in recent years, it has been observed that a delay in treatment impacts survival free of amputation and some kind of complications, is necessary to offer the appropriate treatment in a short period of time. Objective: To determine if in the patients with a diagnosis of vascular trauma attended initially in the HGM compared to those in which their first level of care was a reference hospital, they presented fewer amputations or some other complication. Material and methods: The records of the patients treated in the HGM with the diagnosis of vascular trauma were identified, were divided into 2 groups, the variables age, gender, comorbidities, trauma mechanism, time elapsed between trauma and

Correspondencia:

*Dalid R. Nieto-Zepeda E-mail: dalid.nieto@gmail.com
Disponible en internet: 21-07-2020 Rev Mex Angiol. 2020;48(2):47-52 www.RMAngiologia.com 0377-4740/@ 2020 Sociedad Mexicana de Angiología y Cirugía Vascular y Endovascular, A.C. Published by Permanyer México. This is an open access article under the CC BY-NC-ND license (http://creativecommons.org/licenses/by-nc-nd/4.0/). 
definitive treatment, type of vascular repair, level of amputation, acute renal failure, and mortality. Results: Of the 101 identified patients, 45 files were eliminated, 61 patients were included, there was no difference in terms of demographic variables, it was observed that pulses were not palpable in the exploration in $82 \%$ of the patient's vs $57 \%$ of the patients. referred patients, thrill at admission in $22 \%$ of those referred vs $3 \%$ of those treated in the HGM $(p=0.03)$, thrill at admission in $27 \%$ of those referred vs $3 \%(p=0.01)$. Conclusion: it was observed that in the referred patients the average time between time and surgery was 7 days, the requirement of fasciotomies in these patients increased as well as an increase in surgical times, so it is proposed to make an early referral to a third level center.

Key words: Vascular trauma. Complications. Prompt treatment.

\section{Introducción}

Los traumas vasculares son una patología compleja que puede ocasionar daño arterial y/o venoso, con posterior repercusión al ocasionar desde hemorragias masivas o isquemia aguda, hasta fístulas arteriovenosas o pseudoaneurismas, entre otras complicaciones $^{1,2}$. Es una entidad frecuente desde inicios del siglo XX debido a los distintos conflictos bélicos, y en la actualidad debido al incremento del uso de armas de alta energía, y se encuentra una prevalencia del 50$70 \%$ en la guerra de Irak $^{3}$. Sin embargo, los protocolos de diagnóstico y tratamiento han ido evolucionando a lo largo de los años, y han disminuido las tasas de amputación. Durante la Segunda Guerra Mundial, los pacientes eran tratados con ligadura vascular y presentaban tasas de amputación del $48.9 \%$. Con la aparición de nuevas técnicas y el uso de injertos autólogos, las tasas de amputación en las guerras de Corea y Vietnam disminuyeron hasta un $13 \%$, y mejoraron aún más en la guerra de Irak, con tasas de amputación de 5-10\% ${ }^{4}$. Durante la guerra de Irak, el grupo de Gifford, et al. encontraron que las lesiones por arma de fuego de alta energía, el retraso en el tratamiento de lesiones venosas y la presencia de fracturas concomitantes aumentaban el riesgo de amputación ${ }^{5,6}$. Las lesiones venosas se resolvían en la antigüedad mediante ligadura de las mismas, y presentaban altas tasas de trombosis venosas e, incluso, casos de tromboembolia pulmonar. Brusov, et al. consideran que el reparo de toda lesión venosa es necesario, ya que ayuda a disminuir el edema posquirúrgicos y la permeabilidad arterial a largo plazo ${ }^{7,8}$. Gifford, et al. encontraron que la reparación venosa temprana ayuda también a disminuir la tasa de amputación ${ }^{9}$.

Aproximadamente el $33 \%$ de las lesiones vasculares se encuentran de manera concomitante con fracturas y el $17 \%$, con lesión nerviosa, lo que convierte a las fracturas en un factor independiente para aumentar el riesgo de amputación ${ }^{10}$. De manera tradicional, las fracturas se trataban de manera inicial, y le seguía la reparación arterial, para evitar daño por manipulación ósea; sin embargo, se ha observado un incremento en las tasas de amputación por el mayor tiempo de isquemia, por lo que actualmente se recomienda la reparación vascular primaria, con posterior manejo de la fractura y del daño nervioso $0^{11,12}$.

La arteriografía actualmente se considera el «gold-standard» para el diagnóstico de lesión vascular, identifica el tipo, la localización, su extensión y la presencia o no de colaterales ${ }^{13}$. Sin embargo, por ser un método invasivo y de difícil localización, en algunos centros hospitalarios, se aconseja reservarlo para casos de dificultad diagnóstica con métodos no invasivos ${ }^{14}$.

El ultrasonido Doppler es un método no invasivo, seguro, reproducible y de fácil acceso, que logra observar velocidades y ondas de flujo en tiempo real, así como identificar pseudoaneurismas, disecciones y fistulas arteriovenosas ${ }^{15}$. Se ha observado una precisión del $98 \%$ para el diagnóstico de lesión vascular con uso de ultrasonido Doppler, sin embargo, el hecho de tratarse de un estudio dependiente del operador puede llegar a presentar una sensibilidad de $50 \%$ en algunos centros ${ }^{16}$.

En 1897, Murphy, et al. fueron los primeros en demostrar que las lesiones vasculares podían ser reparadas; sin embargo, no fue hasta la década de 1950, posterior a la Segunda Guerra Mundial, que se observó la tasa de amputación con reparación vascular del $35.8 \%$ vs. una tasa del $49 \%$ en la ligadura, lo que enfatiza la necesidad y beneficio de la reparación vascular ${ }^{17}$. Actualmente existe una tasa de salvamento de la extremidad del $95 \%$; incluso lesiones de arteria poplítea, las cuales previamente se asociaban a una alta tasa de amputación, ahora tienen una tasa de salvamento de $90 \%$, siempre que se realice en las primeras 6-8 horas posteriores al trauma ${ }^{18}$. El trauma vascular se ha incrementado de manera exponencial en los últimos años en nuestro país, y el Hospital General de México (HGM) es un centro de referencia no solo de la ciudad de México, sino también a nivel nacional. En muchas ocasiones, los pacientes son referidos en 
etapas tardías, por lo que prácticamente es imposible realizar algún procedimiento de revascularización y el desenlace final es la pérdida de la extremidad; por tal motivo, el objetivo de nuestro trabajo fue determinar si los pacientes con diagnóstico de trauma vascular atendidos de manera inicial en el HGM, comparados con aquellos en los que su primer nivel de atención fue un hospital de referencia, presentaron menor número de amputaciones o alguna otra complicación.

\section{Métodos}

Presentamos un estudio de cohorte retrospectiva, la cual se llevó a cabo en el Servicio de Angiología y Cirugía Vascular del HGM, donde se incluyeron a todos aquellos pacientes que ingresaron al HGM con el diagnóstico de trauma vascular del 1 de enero del 2013 al 31 de diciembre del 2017, y se dividieron en dos grupos, el primer grupo estuvo integrado por aquellos pacientes atendidos de un inicio en el HGM, y el segundo grupo estuvo integrado por aquellos pacientes referidos de otra unidad hospitalaria, es decir, que ya habían recibido algún otro tipo de manejo, ya sea médico o quirúrgico. Después de haber identificado a los pacientes se revisaron los expedientes clínicos, donde se analizaron las variables como edad, género, comorbilidades, tabaquismo, fecha de ingreso al hospital, tiempo transcurrido entre el trauma y la atención primaria, tiempo transcurrido entre el trauma y la revisión por el servicio de cirugía vascular, tiempo que tardó el paciente en recibir tratamiento quirúrgico desde el trauma y hasta su manejo definitivo, y analizamos también las complicaciones que se presentaron en ambos grupos de pacientes, evaluamos la sobreviva libre de amputación y las diversas complicaciones, como síndrome compartimental, sangrado mayor, falla renal aguda y mortalidad. Se eliminaron del estudio a aquellos pacientes que no reunían los criterios de inclusión o que el expediente clínico se encontraba incompleto.

Una vez recabados todos los datos se realizó un análisis de regresión logística utilizando el programa estadístico SPSS 20.0. Se reportan análisis de las variables con Chi cuadrado.

\section{Resultados}

Se analizaron los expedientes de 106 pacientes, de los cuales 45 fueron eliminados del estudio por no cumplir con los criterios de inclusión o por encontrarse los expedientes clínicos incompletos. De los 61 pacientes que se incluyeron en el estudio, el $80 \%$ fueron del género masculino, con una media de edad entre los $20 \mathrm{y}$

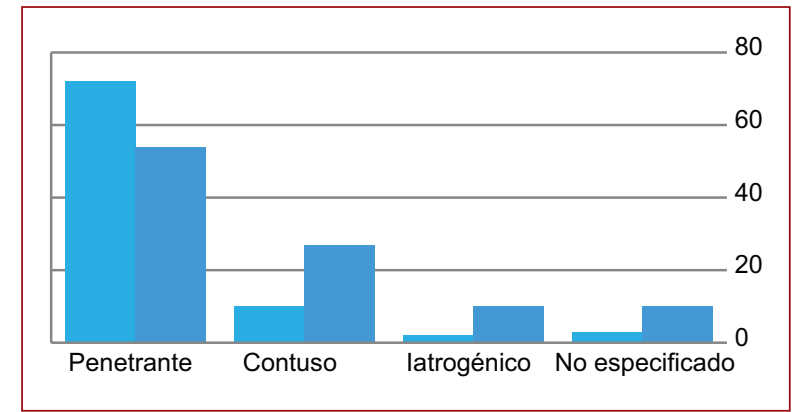

Figura 1. Gráfica del tipo de traumatismo vascular observado en este estudio.

29 años para los pacientes tratados en el HGM y de 30 a 39 años para los pacientes referidos de otros hospitales. No se encontraron comorbilidades preexistentes en los pacientes. En cuanto al tabaquismo, se observó que el $56.7 \%$ de los pacientes atendidos en el HGM no fumaba, comparado con el $27.3 \%$ de los pacientes referidos $(p=0.30)$. En referencia al alcoholismo, el $66 \%$ de la población atendida en el HGM refirió alcoholismo positivo, comparado con el $72 \%$ de los pacientes referidos. En cuanto al mecanismo del trauma fue punzocortante en el $26 \%$ de los pacientes atendidos en el HGM vs. en el $27 \%$ de los referidos, herida por proyectil de arma de fuego en el $46 \%$ de los pacientes del HGM vs. en el $27 \%$ de los referidos, secundario a trauma contuso en el $10 \%$ de los pacientes del HGM vs. en el $27 \%$ de los referidos, trauma de origen iatrogénico en el $2 \%$ de los pacientes del HGM vs. en $10 \%$ de los referidos. En el $3 \%$ de los pacientes atendidos en el HGM de primera vez no se refirió el mecanismo del trauma comparado con el $10 \%$ de los pacientes referidos de otra unidad hospitalaria. Al realizar el análisis estadístico en cada una de estas variables no encontramos diferencia estadística significativa (Fig. 1). Se analizó, en ambos grupos, la localización más frecuente de la lesión vascular, y se identificó que, en los pacientes atendidos en el HGM como primera opción, la localización más frecuentemente encontrada fue en miembros pélvicos, en un $66 \%$, comparada con el $45 \%$ en aquellos pacientes que fueron referidos; el segundo lugar en frecuencia fueron las extremidades superiores, en un $16 \%$ de los atendidos en el HGM, comparado con $45 \%$ de los pacientes referidos; el tercer lugar de frecuencia fue ocupado por trauma del cuello, en un 10\% para los pacientes atendidos en el HGM, comparado con un $4.5 \%$ de los pacientes referidos. Con menor frecuencia atendimos a pacientes con trauma de tórax, abdomen y pelvis, sin que estos tuvieran significancia estadística (Fig. 2). Al 


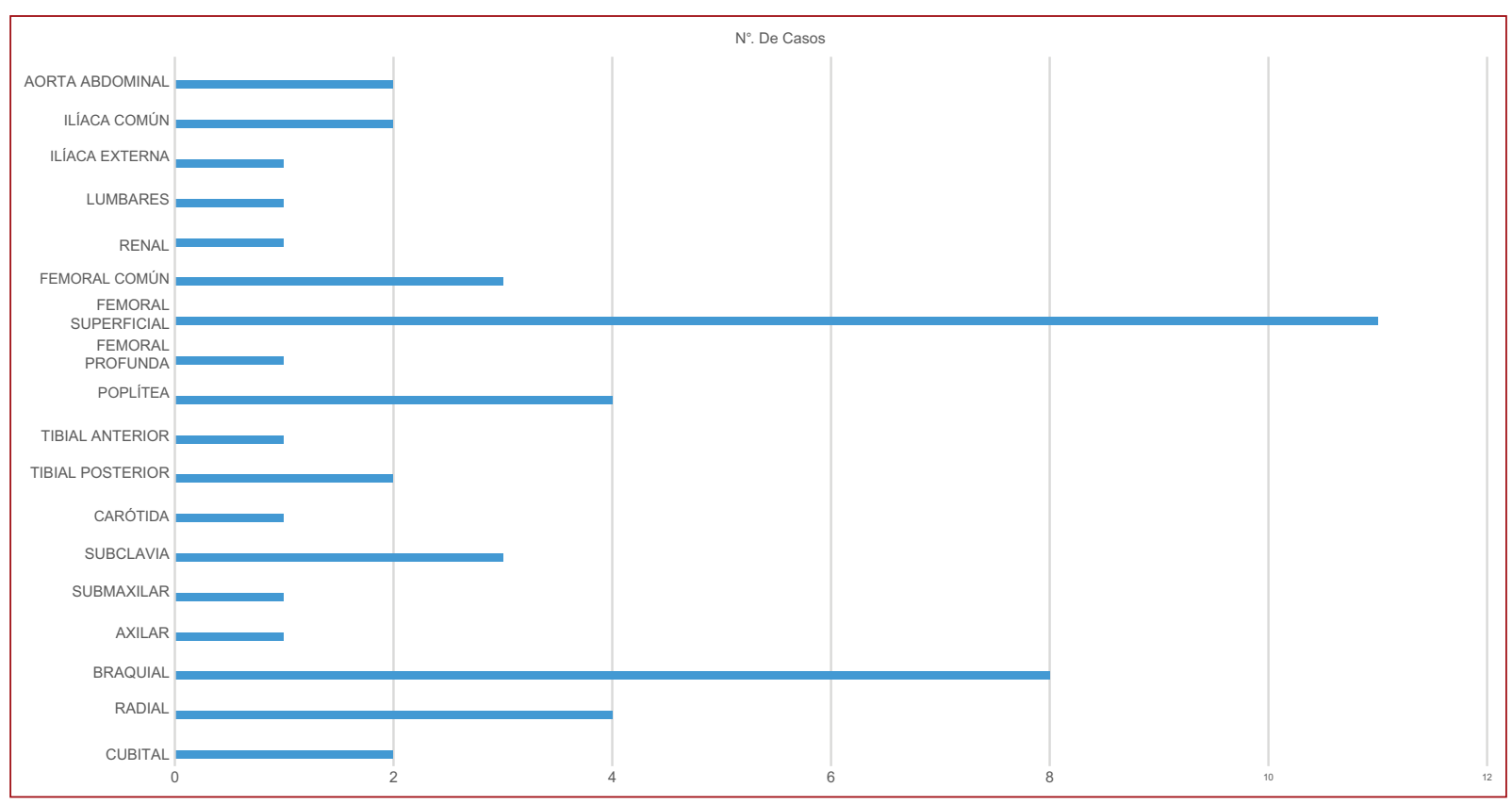

Figura 2. Arterias más frecuentes afectadas por traumatismo vascular.

analizar diversas variables encontramos que el $82 \%$ de los pacientes ingresados de manera inicial en el HGM no tenían pulsos palpables, comparado con el $57 \%$ de los pacientes referidos (esto último reportado en las hojas de referencia) $(p=0.52)$; el $16 \%$ de los pacientes que ingresaron a urgencias del HGM contaban con un hematoma pulsátil comparado con el $32 \%$ de los referidos ( $p=0.03$ ); a la exploración física se documentó soplo en un $3 \%$ de los atendidos en el HGM, comparado con el $22 \%$ de los pacientes referidos ( $p=0.03$ ); se registró thrill al ingreso en el $3 \%$ de la población atendida en el HGM, comparado con el $27 \%$ de los pacientes referidos ( $p=0.01$ ); palidez en el $36 \%$ y el $47 \%$, respectivamente, hipotermia al ingreso en el $20 \%$ y el $27 \%$, dolor al ingreso en 71 y $86 \%$, fractura asociada en $3 \%$ y $9 \%$, antecedente de sangrado activo al ingreso a las respectivas unidades hospitalarias de $34 \%$ en los pacientes del HGM y $59 \%$ en los pacientes referidos, y por ultimo déficit neurológico en ningún paciente de los atendidos en el HGM y en el $9 \%$ de los pacientes referidos. Todas estas variables sin presentar significancia estadística al realizar el análisis.

Cuando se analizó el tiempo en días desde el ingreso hasta la intervención quirúrgica definitiva, se observó que los pacientes ingresados en el HGM se operaron el mismo día, comprado con los pacientes referidos que se operaron en promedio 7 días después del trauma $(p=$

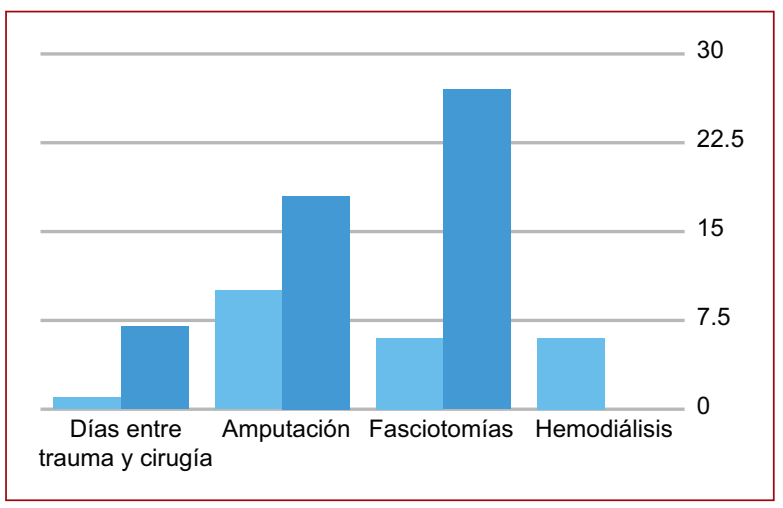

Figura 3. Comparación de las complicaciones entre los grupos de estudio.

0.001). Analizamos las complicaciones posquirúrgicas en ambos grupos, el 10\% tratados de manera inicial en el HGM tuvieron una amputación, comparado con el 18\% de los pacientes referidos. En el $100 \%$ de los pacientes amputados que ingresaron de manera inicial en el HGM se realizó una amputación supracondílea, comprada con el $75 \%$ de los casos referidos, y en un $25 \%$ de realizo una amputación transhumeral. En cuanto a la necesidad de fasciotomías, el $6 \%$ de los pacientes tratado de un inicio en el HGM requirió de las mismas comparado con el $27 \%$ de los pacientes referidos de otras unidades 
hospitalarias $(p=0.04)$. En referencia a la necesidad de hemodiálisis, esta fue necesaria en el $6 \%$ de los pacientes tratados de manera inicial en el HGM, sin ser un tratamiento requerido para los pacientes referidos. No hubo significancia estadística en cuanto al ingreso a la unidad de terapia intensiva (UTI). La mortalidad reportada en los pacientes tratados en el HGM fue del $10 \%$, comparada con el $9 \%$ que se presentó en los pacientes referidos de otra unidad hospitalaria (Fig. 3).

\section{Discusión}

De acuerdo a los resultados obtenidos en nuestro estudio y comparados con lo descrito en la literatura, existe un retraso en el diagnóstico y referencia de los hospitales de primer nivel de atención a un servicio de cirugía vascular. El $80 \%$ de nuestra población fue del género masculino, entre la tercera y cuarta décadas de la vida, lo cual corresponde a lo descrito en la literatura: el estudio de Friend, et al. reportó mayor frecuencia entre los 20-29 años, que fue el grupo etario afectado con mayor frecuencia en nuestro estudio ${ }^{19}$.

El mecanismo de trauma atendido con mayor frecuencia fueron los traumas penetrantes y, de estos, el trauma secundario a herida por proyectil de arma de fuego se encontró con mayor frecuencia en ambos grupos. Se han descrito varias series en las que el trauma penetrante es el principal mecanismo atendido ${ }^{20}$. De acuerdo a los resultados obtenidos en nuestro estudio, observamos que los miembros pélvicos han sido los afectados con mayor frecuencia, lo que es similar a lo descrito en la literatura. Las arterias femoral superficial y poplítea son principalmente las más dañadas, entre un $50-60 \%$ de los casos, seguidas de la arteria braquial, en un $30 \%$ de los casos $^{21}$.

Al analizar las variables en cuanto a exploración física se refiere, encontramos que el signo más frecuente fue la ausencia de pulsos, dolor, hipotermia, hematoma pulsátil, soplo, thrill y fractura asociada. Esto se encontró de manera similar a lo descrito en la literatura, donde encontramos que el $90 \%$ de las lesiones vasculares por arma de fuego pueden ser confirmadas mediante exploración física al encontrar sangrado pulsátil, hematoma expansivo, soplo audible, thrill palpable y las «5P» de isquemia (dolor, parestesia, parálisis, ausencia de pulso y palidez), lo cual es indicativo de exploración quirúrgica inmediata. En caso de encontrar signos blandos de lesión vascular, como son sangrado moderado, pulsos periféricos disminuidos, hematoma estable, trayecto vascular o compromiso neurológico, podemos apoyarnos en la valoración del índice tobillo-brazo, el cual nos indicará alguna alteración en el flujo si se encuentra por debajo de 0.9 , por lo que se tendrán que realizar otros estudios diagnósticos ${ }^{22}$.

Al analizar el tiempo transcurrido entre que se produjo el trauma y que el paciente recibió atención por un cirujano vascular, fue en menos de 6 horas en los pacientes atendidos en el HGM y en un promedio de 7 días para los pacientes referidos, aunque no tenemos un dato exacto de los pacientes amputados, debido a que muchos se contrarrefieren desde el servicio de urgencias una vez valorados por el servicio de cirugía vascular. Una vez confirmado el diagnóstico es primordial iniciar el manejo en las primeras 6-8 horas posteriores a la lesión, ya que la demora puede desencadenar necrosis, disfunción y amputación. Actualmente se encuentran discrepancias en el tiempo ventana para evitar secuelas posteriores. En algunas series se ha mencionado que 2 horas de isquemia pueden ocasionar daño nervioso permanente y que de 4 a 6 horas, muerte muscular con posterior invalidez; sin embargo, Rush, et al. hacen hincapié en que el tejido muscular y nervioso son capaces de tolerar isquemia durante 6 a 8 horas, y consideran este tiempo como ventana. En presencia de inestabilidad hemodinámica durante la cual los pacientes no puedan tolerar algún tipo de procedimiento invasivo mayor, se sugiere la realización de algún tipo de shunt con los distintos materiales existentes, lo cual nos puede llegar a permitir un retraso de 24 e incluso hasta 48 horas para realizar el procedimiento definitivo de revascularización ${ }^{23}$.

En cuanto a las complicaciones postoperatorias, se dieron en el $10 \%$ de los pacientes atendidos en el HGM comparado con el $18 \%$ de los pacientes referidos de otras unidades hospitalarias, si bien la sobreviva libre de amputación fue del $82 \%$ en los pacientes referidos. En 1897, Murphy, et al. fueron los primeros en demostrar que las lesiones vasculares podían ser reparadas; sin embargo, no fue hasta la década de 1950, posterior a la Segunda Guerra Mundial, cuando se observó la tasa de amputación con reparación vascular del $35.8 \%$ vs. una tasa del $49 \%$ en ligadura, lo que enfatizó la necesidad y beneficio de la reparación vascular ${ }^{22}$. Actualmente existe una tasa de salvamento de la extremidad de $95 \%$, incluso lesiones de arteria poplítea, que previamente se asociaban a una alta tasa de amputación, ahora tienen una tasa de salvamento del $90 \%$, siempre que se realice en las primeras 6-8 horas posteriores al trauma ${ }^{24}$. En nuestro estudio encontramos que los pacientes con criterios de irreversibilidad fueron contrarreferidos a sus unidades hospitalarias para la amputación, por lo que no se contó con el expediente físico y, por lo tanto, fueron eliminados del estudio, lo que podría considerarse una debilidad en nuestro estudio. 
En los pacientes referidos de otras unidades hospitalarias se observó la necesidad de realizar fasciotomías con mayor frecuencia que en los pacientes atendidos de primera instancia en el HGM, y no se encontró diferencia en cuanto a requerimientos de hospitalización en la unidad de terapia intensiva o de mortalidad, y tampoco se encontró significancia estadística en cuanto al tipo de reparación o tratamiento quirúrgico, ya sea con el de injerto autólogo o sintético. Coincidimos con la experiencia del Centro Médico Nacional La Raza en que la estrategia terapéutica se basa en el tratamiento quirúrgico precoz y oportuno para un resultado óptimo ${ }^{25}$. Parte importante de la reparación vascular es la resección de los bordes vasculares; algunos autores recomiendan necesario la resección de $1 \mathrm{~cm}$ de borde en casos que hayan sido ocasionados por armas de alta velocidad, y aproximadamente de $3 \mathrm{~mm}$ en caso de heridas ocasionadas por armas de baja velocidad, para evitar daño microscópico que pueda intervenir en la adecuada reparación del vaso, sin embargo, hay discrepancia entre series. Nanobashvili, et al. enfatizan la necesidad de resecar $1 \mathrm{~cm}$ del borde para asegurar una anastomosis de buena calidad ${ }^{26}$. En cuanto al tipo de reparación vascular va a depender del caso a tratar teniendo múltiples opciones desde reparación primaria, uso de parches autólogos y sintéticos, realización de bypass e, incluso, procedimientos endovasculares con uso de stents recubiertos 0 incluso embolización ${ }^{17}$.

\section{Conclusiones}

Se observó que, en los pacientes referidos, el promedio de tiempo entre el trauma y la atención fue de 7 días. Se incrementó el requerimiento de fasciotomías en estos pacientes, así como un incremento en los tiempos quirúrgicos, por lo que se propone realizar una referencia temprana a un centro de tercer nivel.

\section{Conflicto de intereses}

Los autores declaran no tener ningún conflicto de intereses.

\section{Responsabilidades éticas}

Protección de personas y animales. Los autores declaran que para esta investigación no se han realizado experimentos en seres humanos ni en animales.

Confidencialidad de los datos. Los autores declaran que han seguido los protocolos de su centro de trabajo sobre la publicación de datos de pacientes.
Derecho a la privacidad y consentimiento informado. Los autores han obtenido el consentimiento informado de los pacientes y/o sujetos referidos en el artículo. Este documento obra en poder del autor de correspondencia.

\section{Bibliografía}

1. Chong VE, Lee WS, Miraflor E, Victorino GP. Applying peripheral vascular injury guidelines to penetrating trauma. J Surg Res. 2014;190(1): 300-4.

2. Hornez E, Boddaert G, Ngabou UD, Aguir S, Baudoin Y, Mocellin N, et al Temporary vascular shunt for damage control of extremity vascular injury: A toolbox for trauma surgeons. J Visc Surg. 2015;152(6):363-8.

3. Biagioni RB, Miranda GC, Mota de Moraes L, Nasser F, Burihan MC, Ingrund JC. Femoral vessel injury by a nonlethal weapon projectile. $J$ Vasc Surg Cases Innov Tech. 2018;4(2):175-7.

4. Mathew S, Smith BP, Cannon JW, Reilly PM, Schwab CW, Seamon MJ. Temporary arterial shunts in damage control. J Trauma Acute Care Surg. 2017;82(3):512-7.

5. Xu Y, Xu W, Wang A, Meng H, Wang Y, Liu S, et al. Diagnosis and treatment of traumatic vascular injury of limbs in military and emergency medicine. Medicine (Baltimore). 2019;98(18):e15406.

6. Brusov PG, Nikolenko VK. Experience of treating gunshot wounds of large vessels in Afghanistan. World J Surg. 2005;29(Suppl. 1):25-9.

7. Gifford SM, Aidinian G, Clouse WD, Fox CJ, Porras CA, Jones WT, et al. Effect of temporary shunting on extremity vascular injury: An outcome analysis from the Global War on Terror vascular injury initiative. J Vasc Surg. 2009;50(3):549-56.

8. Evans C, Chaplin T, Zelt D. Management of Major Vascular Injuries: Neck, Extremities, and Other Things that Bleed. Emerg Med Clin North Am. 2018;36(1):181-202.

9. Johansen K, Daines M, Howey T, Helfet D, Hansen S. Objective criteria accurately predic amputation following lower extremity trauma. The journal of trauma; 1990;30(5):568-73

10. Ly TV, Travison TG, Castillo RC, Bosse MJ, MacKenzie EJ. Ability of lower-extremity injury severity scores to predict functional outcome after limb salvage. J Bone Jt Surg Ser A. 2008;90(8):1738-43.

11. Scerbo MH, Holcomb JB, Taub E, Gates K, Love JD, Wade CE, et al. The trauma center is too late: Major limb trauma without a pre-hospital tourniquet has increased death from hemorrhagic shock. J Trauma Acute Care Surg. 2017;83:1165-72.

12. Gümbel D, Naundorf M, Napp M, Ekkernkamp A, Seifert J. Diagnostik und Management peripherer Gefäßverletzungen. Unfallchirurg. 2014;117(5):445-60.

13. Pereira BMT, Chiara O, Ramponi F, Weber DG, Cimbanassi S, De Simone $B$, et al. WSES position paper on vascular emergency surgery. World J Emerg Surg. 2015;10(1):1.

14. Marcia L, Kim DY. Predictors of Peripheral Vascular Injury in Patients with Blunt Lower Extremity Fractures. Ann Vasc Surg. 2019;57:35-40.

15. Kuwahara JT, Kord A, Ray CE. Penetrating Extremity Trauma Endovascular versus Open Repair? Semin Intervent Radiol. 2020;37(01):055-61.

16. Bradley MJ, Scalea TM. Violence, Trauma, and Trauma Surgery. Siegler M, Rogers SO, editors. Cham: Springer International Publishing. 1a. Edición, Chicago, EUA, 2020, pp. 223-235.

17. Murphy J. Resection of arteries and veins injured in continuity: End-toend suture: Experimental and clinical research. Med Rec. 1897;(51):73.

18. Dua A, Desai SS, Shah JO, Lasky RE, Charlton-Ouw KM, Azizzadeh A, et al. Outcome predictors of limb salvage in traumatic popliteal artery injury. Ann Vasc Surg. 2014;28(1):108-14

19. Friend J, Rao S, Sieunarine K, Woodroof P. Vascular trauma in Western Australia: a comparison of two study periods over 15 years. ANZ J Surg. 2016;86(3):173-8.

20. Perkins ZB, De'Ath HD, Aylwin C, Brohi K, Walsh M, Tai NR. Epidemiology and outcome of vascular trauma at a British Major Trauma Centre. Eur J Vasc Endovasc Surg. 2012;44(2):203-9.

21. Inaba K, Aksoy H, Seamon MJ, Marks JA, Duchesne J, Schroll R, et al. Multicenter evaluation of temporary intravascular shunt use in vascular trauma. J Trauma Acute Care Surg. 2016;80(3):359-65

22. Kuwahara JT, Kord A, Ray CE. Penetrating Extremity Trauma Endovascular versus Open Repair? Semin Intervent Radiol. 2020;37(01):055-61.

23. Hafez HM, Woolgar J, Robbs JV. Lower extremity arterial injury: Results of 550 cases and review of risk factors associated with limb loss. J Vasc Surg. 2001;33(6):1212-9.

24. Dua A, Desai SS, Shah JO, Lasky RE, Charlton-Ouw KM, Azizzadeh A, et al. Outcome predictors of limb salvage in traumatic popliteal artery injury. Ann Vasc Surg. 2014;28(1):108-14

25. Muñoz-Vega SL, Bizueto-Rosas H, Cossío-Zazueta A, Serrato-Auld RC, Segoviano-Sandoval. Experiencia del Hospital de Especialidades del Centro Médico Nacional La Raza en pacientes con trauma vascular atendidos inicialmente en otra unidad. Rev Mex Angiol. 2019;47(3):22-9.

26. Nanobashvili J, Kopadze T, Tvaladze M, Buachidze T, Nazvlishvili G. War injuries of major extremity arteries. World J Surg. 2003;27(2):134-9. 